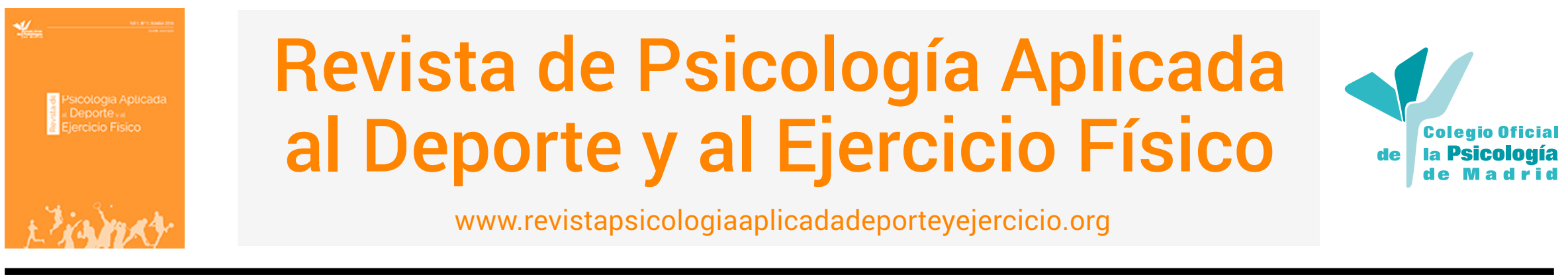

\title{
Formación en relajación para el control de estrés en boxeadores de un Centro de Tecnificación Deportiva
}

\author{
Aurelio Olmedilla \\ Universidad de Murcia, España
}

Isabel Moreno-Fernández

Universidad Autónoma de Barcelona, España

Belén Olmedilla-Caballero

Universidad de Murcia, España

\section{Álvaro Sevilla \\ Universidad Autónoma de Madrid, España}

\section{Verónica Gómez-Espejo \\ Universidad de Murcia, España}

RESUMEN: El estrés es un proceso psicológico que experimentan muchos deportistas en etapas de formación. No sólo puede afectar a su rendimiento deportivo, sino a su calidad de vida y actividades relacionadas con los estudios. Algunas técnicas y habilidades psicológicas pueden resultar muy útiles para el control del estrés. El objetivo de este trabajo es comprobar si las técnicas de relajación son herramientas útiles para controlar el estrés de jóvenes boxeadores y pueden extrapolarse a otros aspectos de su vida como al ámbito académico. Los participantes fueron 7 deportistas jóvenes del Centro de Tecnificación Deportiva de la Federación de Boxeo, varones e internos en el centro. Se realizó un taller de relajación en 5 sesiones de aproximadamente 50-60 minutos cada una. Se trabajaron la técnica de entrenamiento autógeno de Shultz, la técnica de relajación muscular progresiva de Jacobson y la técnica de respiración. Se evaluó (antes y después del taller) la capacidad de los deportistas para gestionar el estrés y la capacidad para afrontar la presión de la evaluación del rendimiento del deportista. De los 7 boxeadores, 4 mostraron puntuaciones que denotaban un mejor control del estrés y una mejor gestión de la presión debida a su rendimiento. Todos mostraron un grado muy alto de satisfacción con el taller. Se concluye que la implementación de un taller de relajación puede ser muy útil para el aprendizaje de técnicas de relajación que ayuden a controlar el estrés, tanto en el ámbito deportivo como en el ámbito académico.

PALABRAS CLAVES: estrés, boxeo juvenil, técnicas de relajación

\section{Training in relaxation for stress control in boxers from an Athlete Training Center}

ABSTRACT: Stress is a psychological process that manyathletes experience in training stages. Not only can it affect your athletic performance, but also your quality of life and sacademic activities. Some psychological techniques and skills can be very helpful in managing stress. The

Agradecimientos/Financiación: Este estudio se ha realizado dentro del Contrato/Programa entre el Centro de Tecnificación Deportiva (CTD) de la Comunidad Autónoma de la Región de Murcia y la Universidad de Murcia (UMU31047-GINVEST10294).

Aurelio Olmedilla Zafra es psicólogo en la Universidad de Murcia.

Isabel María Moreno-Fernández es psicóloga en la Universidad Autónoma de Barcelona.

Belén Olmedilla-Caballero es psicóloga en la Universidad de Murcia.

Álvaro Sevilla es psicólogo en la Universidad Autónoma de Madrid.

Verónica Gómez-Espejo es psicóloga en la Universidad de Murcia.

La correspondencia sobre este artículo debe enviarse a Aurelio Olmedilla al mail: olmedilla@um.es 
aim of this study is to verify whether relaxation techniques are useful tools to control stress in young boxers and can be extrapolated to other aspects of their lives such academic activities. The participants were 7 young athletes from the Athlete Training Center of the Boxing Federation, male and trainees at the center. A relaxation workshop was conducted in 5 sessions of approximately 50-60 minutes each. The Shultz autogenic training technique, Jacobson's progressive muscle relaxation technique and the breathing technique were used. The athletes' ability to manage stress and the ability to cope with the pressure of the athlete's performance evaluation were evaluated (before and after the workshop). Of the 7 boxers, 4 showed scores that denoted better stress control and better pressure management due to their performance. All showed a very high degree of satisfaction with the workshop. It is concluded that the implementation of a relaxation workshop can be very useful for learning relaxation techniques that help control stress, both in sports and academic activities.

KEYWORDS: stress, youth boxing, relaxation techniques

\section{Formação em relaxamento para controlo do stress em boxeadores do Centro de Tecnificação Desportiva}

RESUMO: O stress é um processo psicológico que muitos atletas vivenciam nas etapas de formação. Não só pode afetar o seu rendimento desportivo, mas também a sua qualidade de vida e atividades relacionadas com os estudos. Algumas técnicas e competências psicológicas podem ser muito úteis no controlo do stress. O objetivo deste trabalho é verificar se as técnicas de relaxamento são ferramentas úteis para controlar o stress em jovens boxeadores e se podem ser extrapoladas para outros aspetos das suas vidas, como o âmbito académico. Os participantes foram 7 jovens atletas jovens do Centro de Tecnificação Desportiva da Federação de Boxe, do sexo masculino e internos do centro. Um workshop de relaxamento foi realizado em 5 sessões, de aproximadamente 50-60 minutos cada. Foi utilizada a técnica do treino autógeno de Schultz, a técnica de relaxamento muscular progressivo de Jacobson e a técnica de respiração. A capacidade dos atletas de gerir o stress e a capacidade de lidarem com a pressão da avaliação de desempenho do atleta foram avaliadas (antes e depois do workshop). Dos 7 boxeadores, 4 apresentaram resultados que indicavam um melhor controlo do stress e uma melhor gestão da pressão devido ao seu desempenho. Todos demonstraram um alto grau de satisfação com o workshop. Concluiu-se que a implementação de um workshop de relaxamento pode ser muito útil para a aprendizagem de técnicas de relaxamento que ajudem a controlar o stress, tanto no âmbito desportivo como no âmbito académico.

PALABRAS-CHAVE: stress, boxe juvenil, técnicas de relaxamento

Artículo recibido: 16/09/2021 | Artículo aceptado: 26/09/2021

Es un hecho la importancia que entraña el entrenamiento psicológico en el rendimiento del deportista (Brown y Fletcher, 2017; Buceta, 1998; Connaughton et al., 2010; McCormick et al. 2018), motivo por el cual se ha ido incorporando paulatinamente este trabajo psicológico en los entrenamientos deportivos, tanto individuales como colectivos, normalmente enmarcado en la terapia cognitivo-conductual (Lindern, 2016; Moreno-Fernández et al. 2019; Mostaan et al. 2015; Olmedilla et al. 2017). Sin embargo, hay que tener presente que las habilidades psicológicas, al igual que el ejercicio físico, requieren de un entrenamiento sistemático para que el deportista pueda obtener su nivel de rendimiento óptimo (Dongoran et al. 2019; López et al. 2012; Olmedilla y Dominguez-Igual, 2016; Olmedilla et al., 2018).

En los deportes de combate, y específicamente en el boxeo, el atleta debe reaccionar, ajustar y ejecutar los movimientos técnico-tácticos de la forma más rápida posible, lo que conlleva una importante exigencia en cuanto a los procesos psicológicos: atención-concentración, razonamiento o memoria (Suárez-Rodríguez et al. 2018). Sabiendo esto, en el caso del boxeo, se han llevado a cabo numerosas investigaciones explorando específicamente diversos aspectos, como los nive- les de ira (Menéndez y Fernández-Río, 2015; Oliva et al. 2012), la concentración (Cañizares et al. 2020), la motivación (Romero et al. 2019), el afrontamiento de las transiciones (Schinke et al. 2015) o las habilidades psicológicas más significativas que poseen los deportistas según la disciplina (Dongoran et al. 2019). Además, también se han llevado a cabo diversas intervenciones psicológicas en deportes de combate (Emara, 2012; Olmedilla-Caballero et al., 2020; Zamora-Martínez et al., 2017). El trabajo de Zamora-Martínez et al. (2017), muestra una intervención con un luchador de la selección española de lucha grecorromana para disminuir sus niveles de ansiedad precompetitiva. El trabajo de Olmedilla-Caballero et al. (2020) muestra la intervención psicológica realizada con un deportista paralímpico de taekwondo de cara a preparar los Juegos Paralímpicos mientras se recuperaba de una lesión.

Las intervenciones en boxeo no han ido únicamente destinadas a deportistas de élite, sino también a deportistas en edades formativas o juveniles, explorando cómo influyen diversas variables psicológicas en los boxeadores jóvenes y formas de aumentar, disminuir o mantenerlas, como la estimulación del control emocional (Infante-Barzaga, 2016), el aumento de la atención-concentración (Suárez-Rodríguez et 
al. 2018), el bienestar emocional (Minot et al. 2019) o el estrés (Dallmann et al. 2016; Rumbold et al. 2012).

Cuando se habla de deportistas jóvenes, no se debe obviar la importancia de la carrera dual (DC) y la necesidad de que ésta esté equilibrada, permitiendo así que el deportista pueda cumplir tanto sus objetivos deportivos como académicos sin ver perjudicados ni su salud ni su bienestar (Stambulova et al. 2015). Prestando atención al desarrollo de sus competencias (Dreyfut et al., 2019; Pérez-Rivases et al., 2020), al papel de sus padres/madres (Tessitores et al., 2020), y a otros factores determinantes (autoeficacia, objetivos, identidad deportiva) en las decisiones que los jóvenes deportistas deben tomar para desarrollar una carrera académica simultánea (da Costa et al., 2021; Defruyt et al., 2020; Monteiro et al., 2021). Por tanto, una de las acciones que parecen importantes en este sentido será la de proveer al deportista de técnicas y estrategias para este fin, ya que durante la adolescencia y la juventud la carga de los entrenamientos suele ser alta, al igual que las responsabilidades académicas (Brustio et al., 2020; Conde et al., 2021). Teniendo en cuenta las altas demandas educativas y deportivas, así como la presión por rendir, los conflictos con el entrenador o los compañeros, la presión del público y el miedo a una posible lesión no sorprende que los deportistas jóvenes que tienen una carrera dual reporten un nivel de estrés superior que el resto de su grupo de edad (Dallmann et al. 2016). En relación con esto, Miró-Moyá et al. (2017) se propusieron explorar cuáles eran las competencias que los deportistas de alto rendimiento consideraban más necesarias a la hora de organizar la carrera dual, siendo la "Capacidad para afrontar el estrés en el deporte y los estudios" de las tres más nombradas (22 de 36 ) en el grupo de estudiantes de secundaria. Aunque algunos deportistas son capaces de manejar la amplia gama de demandas del ambiente y presiones psicológicas, que pueden ser causas o consecuencias del proceso del estrés, muchos otros no son capaces de llevarlo a cabo de forma eficaz, lo que supone deficiencias en su desempeño y su salud (Rumbold et al. 2012).

En este sentido, Guirola-Gómez et al. (2018) encontraron que la presión y el estrés son percibidos por remeros de alto rendimiento como la principal barrera psicológica a la hora de compaginar la vida académica con la deportiva, sobre todo cuando se encuentran en época de exámenes o de competición. Los resultados destacaron un papel relevante de los padres en el apoyo a los atletas de DC e información parcial sobre las estrategias de apoyo de los padres. En conclusión, el limitado tamaño de la muestra y la tipología de los deportes, y la representatividad parcial de los países han impactado en la aplicación global de los principales hallazgos. Además, la necesidad de un programa educativo para los padres y la necesidad de un compromiso regular entre los padres, los deportistas, el maestro y el entrenador se consideraron cruciales para facilitar las intervenciones exitosas de los padres a nivel académico y / o deportivo y para limitar los posibles efectos negativos de la crianza de los hijos en DC.

Por tanto, el objetivo de este trabajo es presentar la implementación de un taller de relajación realizado con boxeadores jóvenes, y determinar si las técnicas de relajación son percibidas por los jóvenes boxeadores como herramientas útiles para el control del estrés tanto en el ámbito deportivo como en otros ámbitos extradeportivos, como el académico. La hipótesis de trabajo es que el aprendizaje de las técnicas de relajación del taller será percibido por los jóvenes boxeadores como útil para el control del estrés tanto en el ámbito deportivo como en otros ámbitos, como el académico.

\section{Método}

\section{Participantes}

Los participantes fueron 7 deportistas jóvenes del CAR de la Federación de Boxeo, todos varones e internos en el centro, con unas 5 horas de entrenamiento al día 5 días a la semana. Participan en competiciones regionales, nacionales e internacionales. En la Tabla 1 se pueden observar las características sociodemográficas y deportivas más relevantes.

Tabla 1. Características sociodemográficas y deportivas de los deportistas

\begin{tabular}{cclcccl}
\hline Sujeto & Edad & Localidad procedencia & Categoría & Experiencia (años) & Estudios & Competiciones \\
\hline 1 & 16 & Galdácano (Euskadi) & Junior & 5 & $1^{0}$ Bach & Nacionales \\
\hline 2 & 15 & Parla (Madrid) & Junior & 7 & $4^{0}$ ESO & Nacionales \\
\hline 3 & 17 & Córdoba (Andalucía) & Junior & 5 & $2^{0}$ Bach & Internacionales \\
\hline 4 & 16 & Murcia & Junior & 3 & $1^{0}$ Bach & Nacionales \\
\hline 5 & 16 & SP Pinatar (Murcia) & Junior & 3 & $1^{0}$ Bach & Nacionales \\
\hline 6 & 16 & San Javier (Murcia) & Junior & 3 & $1^{0}$ Bach & Nacionales \\
\hline 7 & 16 & SP Pinatar (Murcia) & Junior & 3 & $1^{0}$ Bach & Nacionales \\
\hline
\end{tabular}




\section{Instrumentos}

Se evaluó la capacidad de los deportistas para gestionar el estrés propio de entrenamientos y competición y la capacidad para afrontar la presión de la evaluación del rendimiento del deportista (de los otros y de él mismo). Para ello se utilizaron las escalas Control de Estrés (CE) e Influencia de la Evaluación del Rendimiento (IER) del Cuestionario Características Psicológicas Relacionadas con el Rendimiento Deportivo (CPRD) de Gimeno et al. (2001). El CPRD es un cuestionario de auto-informe formado por 55 ítems de escala tipo Likert, con 5 alternativas de respuesta en un rango de 0 (totalmente en desacuerdo) a 4 (totalmente de acuerdo). La escala CE tiene un alfa de Cronbach de .88, y la escala IER de .72. Como aparece descrito en Gimeno et al. (2001) este cuestionario cuenta con los campos descriptivos necesarios para la notación de sexo, edad, número de años practicados, categoría, deporte y club. Por otro lado, también se evaluó, al finalizar el programa, la satisfacción y la eficacia percibida del programa por parte de los deportistas (ad hoc), aspecto de gran relevancia para poder conocer la opinión de los propios deportistas sobre su proceso de aprendizaje de los contenidos del taller, así como la viabilidad de la aplicación de las técnicas aprendidas.

\section{Procedimiento}

El taller de relajación se realizó en 5 sesiones de 50-60 minutos cada una, y fue dirigido por una psicóloga con una experiencia de 3 años como alumna interna en Psicología del Deporte, habiendo realizado prácticas en el Centro de Tecnificación Deportiva (CTD) "Infanta Cristina" en los Narejos (Los Alcázares).

En la Tabla 2 se muestra el cronograma y el contenido de las sesiones que se llevaron a cabo. Dentro de la planifi- cación del trabajo psicológico con los deportistas del CTD se programaron diferentes acciones.

El taller de relajación para los boxeadores se propuso a éstos y a su entrenador (responsable de la Federación de Boxeo dentro del CTD). En una breve reunión previa con ambos se les explicó en qué consistía el taller y los objetivos perseguidos. De esta manera, todos los deportistas fueron informados del objetivo del estudio y de la confidencialidad tanto de sus respuestas como de los datos obtenidos posteriormente. Se obtuvo el consentimiento informado de todos los padres/tutores legales de los jugadores ya que éstos eran menores de edad.

En la sesión 1 se explicó a los deportistas qué era la relajación y los beneficios que ésta podría comportar para ellos (Díaz et al., 2012); además se les explicó brevemente las 3 técnicas de relajación que se iban a trabajar. Por último, se suministró el CPRD para su cumplimentación. En la sesión 2 se explicó Técnica de Relajación Autógena de Schultz (Schultz, 1980), consistente en que el deportista autogenere, mediante la concentración continua en sensaciones de frío y calor, de peso y ligereza, de latidos de corazón y de respiración, la relajación en diferentes partes del cuerpo. Se utilizó unos 5 minutos para explicar en qué consiste, y tras ello unos 35 minutos de actividad práctica; se indicó a los deportistas que practicasen la técnica al menos 3 veces antes de la próxima sesión, y se les proporcionó un registro que debían de cumplimentar cada vez que practicasen (esto se hizo para las 3 técnicas). En la sesión 3 se explicó Técnica de Relajación Progresiva de Jacobson (Jacobson, 1938), consistente en tensar y relajar grupos musculares desde la cabeza a los pies para que los sujetos adquieran conciencia de la relajación producida en cada grupo de músculos. Se utilizaron unos 10 minutos para explicar en qué consiste, y tras ello unos 50 minutos de actividad práctica. En la sesión 4 se explicó la Técnica de Respiración (Labrador et al., 1995), utilizando unos 10 minutos para explicar en qué consiste, y tras ello unos 50

Tabla 2. Cronograma y contenidos generales del taller

\begin{tabular}{cccc}
\hline Fecha & Sesión & Contenidos & Duración \\
\hline 4-Nov.-2019 & 1 & Presentación del taller. Evaluación inicial & 50 min. \\
\hline 11-Nov.-2019 & 2 & Técnica de Relajación Autógena de Schultz (explicación y actividad práctica) & 40 min. \\
\hline 18-Nov.-2019 & 3 & Técnica de Relajación Progresiva de Jacobson (explicación y actividad práctica) & 60 min. \\
\hline 25-Nov.-2019 & 4 & Técnica de Respiración (explicación y actividad práctica) & 50 min. \\
\hline 2-Dic.-2019 & 5 & Evaluación final & 90 min. \\
\hline
\end{tabular}


minutos de actividad práctica. Por último, la sesión 5 se estableció como cierre, por lo que en una primera parte de la sesión se hizo un breve resumen de todo lo desarrollado en el taller y una ronda de preguntas por si había dudas o algo que quisieran decir; se les volvió a administrar el CPRD, y se pasó el cuestionario de satisfacción/eficacia sobre el taller.

\section{Resultados}

\section{Evaluación de las características psicológicas relacionadas con el control y gestión del estrés}

En la Tabla 3 se muestran las puntuaciones de los boxeadores al inicio del Taller y al finalizar el mismo respecto al factor CE.

Es necesario destacar que 4 de los 7 boxeadores, muestran puntuaciones más altas después del taller, con diferencias importantes en los deportistas 3 y 1 .
En la Tabla 4 se muestran las puntuaciones de los boxeadores al inicio del Taller y al finalizar el mismo respecto al factor IER.

Al igual que en $\mathrm{CE}$, en IER es de destacar que, de los 7 boxeadores, también 4 muestran puntuaciones más altas después del taller, con diferencias importantes en los deportistas 3 y 1 .

\section{Valoración de la eficacia de la intervención}

En la Tabla 5 se muestra la valoración realizada por los deportistas respecto al taller de relajación (se indican las medias en las respuestas cuantitativas y se exponen los comentarios más relevantes de los deportistas). Se suministró a los deportistas un cuestionario de valoración que evaluaba, tanto cuantitativa como cualitativamente, la eficacia de la intervención, la satisfacción y la aplicabilidad a otros ámbitos (Olmedilla-Caballero et al., 2020).

Como se puede observar en la Tabla 5 la satisfacción con el taller de relajación para los boxeadores es bastante

Tabla 3. Puntuaciones pre-post (directas y centiles) en CE de los deportistas

\begin{tabular}{|c|c|c|c|c|c|}
\hline Sujeto & $\begin{array}{c}\text { Puntuación directa } \\
\text { pre-taller }\end{array}$ & $\begin{array}{c}\text { Puntuación centil } \\
\text { pre-taller }\end{array}$ & $\begin{array}{c}\text { Puntuación directa } \\
\text { post-taller }\end{array}$ & $\begin{array}{c}\text { Puntuación centil } \\
\text { post-taller }\end{array}$ & $\begin{array}{l}\text { Diferencia en } \\
\text { Centiles }\end{array}$ \\
\hline 1 & 38 & 30 & 57 & 80 & +50 \\
\hline 2 & 40 & 60 & 48 & 55 & -5 \\
\hline 3 & 23 & 10 & 70 & 99 & +89 \\
\hline 4 & 44 & 45 & 48 & 55 & +10 \\
\hline 5 & 45 & 50 & 34 & 20 & -30 \\
\hline 6 & 46 & 50 & 45 & 50 & $=$ \\
\hline 7 & 38 & 30 & 49 & 60 & +30 \\
\hline
\end{tabular}

Tabla 4. Puntuaciones pre-post (directas y centiles) en IER de los deportistas

\begin{tabular}{cccccc}
\hline Sujeto & $\begin{array}{c}\text { Puntuación directa } \\
\text { pre-taller }\end{array}$ & $\begin{array}{c}\text { Puntuación centil } \\
\text { pre-taller }\end{array}$ & $\begin{array}{c}\text { Puntuación directa } \\
\text { post-taller }\end{array}$ & $\begin{array}{c}\text { Puntuación centil } \\
\text { post-taller }\end{array}$ & $\begin{array}{c}\text { Diferencia en } \\
\text { Centiles }\end{array}$ \\
\hline 1 & 24 & 50 & 36 & 95 & +45 \\
\hline 2 & 21 & 35 & 21 & 35 & +75 \\
\hline 3 & 15 & 10 & 33 & 85 & -30 \\
\hline 4 & 27 & 65 & 22 & 40 & -30 \\
\hline 5 & 25 & 55 & 23 & 80 & 85 \\
\hline 6 & 29 & 65 & 31 & & +5 \\
\hline 7 & 27 & & 33 & & +20 \\
\hline
\end{tabular}


Tabla 5. Valoración por parte de los deportistas del taller de relajación

¿Estás satisfecho/a con el taller de relajación realizado? (indica de 0 =nada satisfecho, a $10=$ muy satisfecho; $y$ responde las 2 siguientes cuestiones)

Indica los aspectos que más te han gustado y razona porqué

Te enseña a relajarte en momento de tensión

Me ha gustado la forma de explicar los ejercicios y la manera de resolver las dudas; me ha ayudado mucho.

Me ha ayudado a relajarme, me encuentro mejor

Me ha gustado mucho la técnica de Jacobson

El aprender a controlar la respiración para relajarme

Indica los aspectos que menos te han gustado y razona porqué

El horario del taller no me ha gustado; tenía que ser más tarde

El tener que pensar cosas en algún ejercicio de relajación

El tensar el cuello me producía "tiricia"

¿En qué medida crees que te ha servido para tu preparación deportiva general?

Indica los aspectos que más te han gustado y razona porqué

Me ha servido para ayudarme cuando me desconcentro

Sobre todo para relajarme justo antes de una competición

Me ha servido para poder tranquilizarme antes de competir

Indica los aspectos que menos te han gustado y razona porqué

Creo que la respiración diafragmática, quizá porque no sé utilizarla bien

¿En qué medida consideras que te ha servido para la competición deportiva?

Indica los aspectos que más te han servido y razona porqué

Para tranquilizarme la noche antes de la competición

Para afrontar mejor la competición

Ahora ya no me pongo tan nerviosa en las competiciones

Me han servido para relajarme antes de los combates

Indica los aspectos que menos te han servido y razona porqué

Soy muy tranquilo y no suelo utilizar la relajación

El taller de relajación ¿te ha servido para otros ámbitos ajenos al deporte y la competición?

Indica y explica en qué ámbitos te ha servido el taller de relajación realizado

Para quitarme el estrés acumulado por exámenes, problemas familiares y otros

Para afrontar mejor los exámenes

Para los exámenes

Para relajarme en momentos de estrés

Para los estudios y los exámenes

alta (8.71). Los aspectos resaltados por los deportistas son principalmente el aprendizaje de estrategias para auto-relajarse y controlar el estado de tensión. También se puede apreciar que los sujetos valoran que estos conocimientos y habilidades en relajación han servido para su preparación deportiva (7.85) y también para la competición (7.14), destacando aspectos como la reducción de tensión o los "nervios" previos a la competición, focalizar la atención y aumentar la concentración en la actividad deportiva. Por último, se puede observar que también valoran que este taller puede ayudarles o beneficiarles en otros aspectos ajenos al deporte y a la competición (8.33). Los aspectos 
Tabla 6. Valoración de la satisfacción y la eficacia de las técnicas y estrategias utilizadas

\begin{tabular}{|c|c|}
\hline Técnicas & Valoración cuantitativa SATISFACCIÓN (de 0 a 10) \\
\hline Relajación Autógena de Schultz & 8.71 \\
\hline Respiración diafragmática & 7.16 \\
\hline Relajación Progresiva de Jacobson & 8.00 \\
\hline Técnicas & Valoración cuantitativa EFICACIA (de 0 a 10) \\
\hline Relajación Autógena de Schultz & 9.14 \\
\hline Respiración diafragmática & 6.66 \\
\hline Relajación Progresiva de Jacobson & 7.14 \\
\hline Técnicas & $\begin{array}{l}\text { Valoración cualitativa GENERAL } \\
\text { indica lo que consideres más representativo de cada técnica o estra- } \\
\text { tegia; si la has utilizado mucho o no, si te ha resultado sencilla o no, } \\
\text { y cualquier otro aspecto que consideres importante }\end{array}$ \\
\hline Relajación Autógena de Schultz & $\begin{array}{l}\text { Sí, la he utilizado } \\
\text { La he utilizado mucho y me ha resultado sencilla y útil } \\
\text { La utilizo para dormir más rápido }\end{array}$ \\
\hline Respiración diafragmática & $\begin{array}{l}\text { Se aprende a respirar mejor } \\
\text { Se aprende a controlar la respiración } \\
\text { No la he utilizado mucho pero cuando lo he hecho me ha resultado } \\
\text { eficaz } \\
\text { La utilizo para relajarme de vez en cuando }\end{array}$ \\
\hline Relajación Progresiva de Jacobson & $\begin{array}{l}\text { Se aprende a tensar y relajar los músculos } \\
\text { La he utilizado cuando me dolía la cabeza y me ha ayudado }\end{array}$ \\
\hline
\end{tabular}

en los que se ven beneficiados los sujetos son sobre todo en el afrontamiento de pruebas académicas y otro tipo de problemas.

En la Tabla 6 se describen las valoraciones cuantitativas y cualitativas que han realizado los deportistas respecto a las técnicas de relajación del taller.

Como se puede observar, la técnica de Relajación Autógena de Schultz es la que ha sido considerada por los deportistas como más satisfactoria (8.71) y más eficaz (9.14), resaltando aspectos como el ser una técnica muy útil y sencilla. Por otro lado, la técnica de Relajación Progresiva de Jacobson es bastante satisfactoria (8) y también la consideran una técnica eficaz (7.14), resaltando aspectos como que aumenta la conciencia y el control sobre los músculos. Por último, la satisfacción respecto a la técnica de Respiración Diafragmática es alta (7.16), aunque la eficacia que le atribuyen es moderada (6.66), indicando que no la han utilizado con frecuencia, aunque aprenden a respirar mejor.

\section{Discusión}

El objetivo del presente trabajo ha sido presentar la implementación de un taller de relajación realizado con boxeadores jóvenes. Además, determinar si las técnicas de relajación son percibidas por los jóvenes boxeadores como herramientas útiles para el control del estrés tanto en el ámbito deportivo como en otros ámbitos extradeportivos, como el académico. Por ello, la hipótesis de trabajo planteada fue que el aprendizaje de las técnicas de relajación del taller sería percibido por los jóvenes boxeadores como útil para el control del estrés tanto en el ámbito deportivo como en otros ámbitos, como el académico.

De acuerdo con los resultados, el taller tuvo efectos positivos en cuanto al CE y la IER para la mayoría de los boxeadores participantes, factores ambos que se han puesto en relación con la mejora del rendimiento deportivo (Barker et al., 2020; Eysenck et al., 2007; Filaire et al., 2001; Hanton et al., 2007; Zamora-Martínez et al., 2017; Ziv y Lidor, 2013). Se observa que 4 participantes experimentaron un incre- 
mento en la puntuación en CE en la evaluación post-test, 2 sujetos manifestaron una puntuación menor y uno de los deportistas mostró la misma puntuación. Resultados similares se encontraron en la IER, siendo 4 los participantes que incrementaron su puntuación en la medida post-test respecto a la medida pre-test. Parece muy importante tener en cuenta la variabilidad individual en el aprendizaje y posterior aplicación de técnicas aprendidas, que efectivamente pue estar condicionando los resultados pre y post de un taller de este tipo.

La importancia de la intervención con técnicas de relajación radica en la influencia que el estrés puede tener y que supone un impacto negativo en los deportistas, especialmente en su rendimiento deportivo. Por tanto, se hacen necesarias estrategias en forma de técnicas de entrenamiento mental para los deportistas que permitan controlar y gestionar adecuadamente el estrés. Los resultados aquí encontrados están en la línea de lo hallado por otras investigaciones (Olmedilla et al., 2019). El estudio de Connaughton et al. (2010) mostró que en general los deportistas de artes marciales que tuvieron mejor rendimiento mostraron mayor control de estrés que sus oponentes. Esto puede deberse a que los niveles de estrés se asocian con la activación de las neuronas en la amígdala y con la secreción de cortisol y oxitocina (Davis, 1997) y, por tanto, las técnicas de manejo del estrés tienen como objetivo reducir la liberación de estas hormonas del estrés (Li et al., 2020). El trabajo de Olmedilla et al. (2019) mostró una intervención cognitivo-conductual para el control del estrés en jugadores jóvenes de fútbol, que mejoró las habilidades psicológicas de los futbolistas para controlar el estrés.

En cuanto a la valoración de la eficacia de la intervención por parte de los propios participantes, la puntuación media en la satisfacción del taller fue de 8.71 puntos, lo cual pone de manifiesto la buena aceptación hacia el mismo por parte de los boxeadores. Por otra parte, los deportistas, con su valoración, indicaron la eficacia de la intervención en su preparación deportiva general, en la competición deportiva y en otros ámbitos ajenos al deporte y a la competición. En este sentido, en la aplicación del entrenamiento a otros ámbitos hay diferentes estudios que demuestran que la creación de una sólida base de habilidades fruto de un buen entrenamiento mental facilitan el buen dominio y las respuestas de afrontamiento en entornos desafiantes (Pain et al., 2011; Schinke, 2004; Todd et al., 2011); quizá uno de los casos más relevantes sea la fortaleza mental mostrada por deportistas jóvenes durante el confinamiento sufrido a nivel mundial por la pandemia COVID-19 (Leguizamo et al., 2021). Concretamente, la aplicación de técnicas de relajación para el control de estrés, pueden resultar de utilidad ya que facilita el control de la atención, el aumento de la concentración y reduce la ansiedad (Anchique, 2006; Barker et al., 2020; Estrada y Pérez, 2008) lo que permite al sujeto procesar la información de manera más eficaz (Korobeynikov et al., 2013) y centrarse en lo realmente necesario e importante, convirtiéndolo en más exitoso en el terreno académico. Ahondando más en este aspecto, la parte general del rendimiento se construye a través de una consideración de las experiencias de la vida (Schinke, 2004). Finalmente, la relajación autógena de Schultz fue considerada por los deportistas como la técnica de relajación practicada más efectiva y satisfactoria, quizá por la percepción de autorregulación que implica la técnica.

La literatura científica muestra que no existen muchas publicaciones que presenten programas de entrenamiento psicológico específicos y de manera sistemática diseñados para que los deportistas puedan controlar y practicar habilidades mentales que sean útiles para mejorar el rendimiento en boxeadores (Dongoran et al., 2019). Por ello, y atendiendo a los resultados de este trabajo parece oportuno considerar el control de estrés como un factor a tener en cuenta no solo para mejorar el rendimiento deportivo de boxeadores, sino para proveerlos de herramientas que les permitan derribar posibles barreras de su vida en general. Además, ello ayudaría a superar algunas de las limitaciones de estudios como el presente, como el tener un número demasiado pequeño de sujetos analizados.

\section{Referencias}

Anchique, F. (2006). Efectos de la autosugestión positiva en el incremento de la efectividad del servicio en tenistas de campo. Revista Iberoamericana de Psicología del Ejercicio y el Deporte, 7(1), 13-26.

Barker, J.B., Slater, M.J., Pugh, G., Mellalieu, S.D., McCarthy, P.J., Jones, M.V. y Moran, A. (2020). The effectiveness of psychological skills training and behavioural interventions in sport using single-case designs: A meta regression analysis of the peer-reviewed studies. Psychology of Sport and Exercise, 51, Artículo 101746. https://doi.org/10.1016/j.psychsport.2020.101746

Brown, D. y Fletcher, D. (2017). The effects of psychological and psychosocial interventions on sport performance: a meta-analysis. Sports Medicine, 47(1), 77-99.

Brustio, P. R., Rainoldi, A., Mosso, C. O., de Subijana, C. L. y Lupo, C. (2020). Italian student-athletes only need a more effective daily schedule to support their dual career. Sport Sciences for Health, 16(1), 177-182.

Buceta, J. M. (1998). Psicología del entrenamiento deportivo. Dykinson.

Cañizares, M., Soler-Prieto, Y., Domínguez, J. y Suárez-Rodríguez, M. (2020). Los factores que afectan la concentración de la atención en atletas de boxeo en situaciones de combate. Podium. Revista de Ciencia y Tecnología en la Cultura Física, 15(1), 5-21. 
Conde, E., Meroño, L., Arias-Estero, J. L., García-Roca, J. A., Leiva-Arcas, A., Cánovas-Álvarez, F. J., Isidori, E., \& Sánchez-Pato, A. (2021). Percepción de la influencia del modelo Estport en la carrera dual de los estudiantes-deportistas en universidades de España e Italia. Cultura, Ciencia y Deporte, 16(47), 31-37. https://doi.org/10.12800/ccd.v16i47.1623

Connaughton, D., Hanton, S. y Jones, G. (2010). The Development and Maintenance of Mental Toughness in the World's Best performers. The Sport Psychologist, 24(2), 168-193. https://doi. org/10.1123/tsp.24.2.168

da Costa, F. R., Torregrosa, M., Figueiredo, A. y Soares, A. J. G. (2021). Make it easier: A narrative review of dual-career empirical studies. Retos: nuevas tendencias en educación física, deporte y recreación, 41, 104-111.

Dallmann, P., Bach, C., Zipser, H., Thomann, P. A. y Herpertz, S. C. (2016). Evaluation of a stress prevention program for young high-performance athletes. Mental Health \& Prevention, 4(2), 7580. https://doi.org/10.1016/j.mhp.2016.04.001

Davis, M. (1997). Neurobiology of fear responses: the role of the amygdala. The Journal of Neuropsychiatry and Clinical Neurosciences, 9(3), 382-402. https://doi.org/10.1176/jnp.9.3.382

Defruyt, S., Wylleman, P., Kegelaers, J. y De Brandt, K. (2020). Factors influencing Flemish elite athletes' decision to initiate a dual career path at higher education. Sport in Society, 23(4), 660-677

Defruyt, S., Wylleman, P., Torregrossa, M., Schipper-van Veldhoven, N., Debois, N., Cecić Erpič, S. y De Brandt, K. (2019). The development and initial validation of the dual career competency questionnaire for support providers (DCCQ-SP). International Journal of Sport and Exercise Psychology, 1-18.

Díaz, M. I., Villalobos, A. y Ruíz, M. A. (2012). La desensibilización sistemática y técnicas de relajación. En M. Díaz, M. A. Ruíz y A. Villalobos (Eds.). Manual de técnicas de intervención cognitivo-conductuales (pp. 237-276). España: Desclée de Brouwer.

Dongoran, M. F, Nopiyanto, Y. E., Saputro, D. P. y Nugroho, A. I. (2019). Comparison of Psychological Skills of Pencak Silat and Boxing Athletes (Study on Indonesian Training Camp athletes). Advances in Social Science, Education and Humanities Research, 383, 124 - 128. https://doi.org/10.2991/icss-19.2019.210

Dongoran, M. F., Fadlih, A. M. y Riyanto, P. (2019). Psychological characteristics of martial sports Indonesian athletes based on categories art and fight. Enfermería Clínica, 30(4), 500-503. https://doi.org/10.1016/i.enfcli.2019.10.129

Emara, A. (2012). Strategies of mental imagery and it's relation to some psychological variables in sport of wrestling. World Journal of Sport Sciences, 6(2), 167- 172. https://doi.org/10.5829/ idosi.wjss.2012.6.2.1131

Estrada, O. y Pérez, E. (2008). Palabras e imágenes positivas en la respuesta de ansiedad en deportistas de competición. Cuadernos de Psicología del Deporte, 8(1), 31- 45.

Eysenck, M.W., Derakshan, N., Santos, R. y Calvo, M.G. (2007). Anxiety and cognitive performance: Attentional control theory. Emotion, 7(2), 336-353. https://doi.org/10.1037/15283542.7.2.336

Filaire, E., Sagnol, M., Ferrand, C., Maso, F. y Lac, G. (2001). Psychophysiological stress in judo athletes during competitions. Journal of Sports Medicine \& Physical Fitness, 41(2), 263-268.
Gimeno, F., Buceta, J.M. y Pérez-Llantada, M. (2001). El cuestionario "Características psicológicas relacionadas con el rendimiento deportivo" (CPRD): Características psicométricas. Análise Psicológica, 1(19), 93-133. https://doi.org/10.14417/ap.346

Guirola-Gómez, I., Torregrosa, M., Ramis, Y. y Jaenes, J.C. (2018). Remando contracorriente: facilitadores y barreras para compaginar el deporte y los estudios. Revista Andaluza de Medicina del Deporte, 17(1), 12-17.

Hanton, S., Cropley, B., Neil, R., Mellalieu, S.D. y Miles, A. (2007). Experience in sport and its relationship with competitive anxiety. Experience in Sport, 5(1), 28-53. https://doi.org/10.1080/1612197X.2008.9671811

Infante-Barzaga, C. M. (2016). El autocontrol emocional en atletas de boxeo. Revista Cubana de Medicina del Deporte y la Cultura Física, 17(1).

Jacobson, E. (1938). Progressive relaxation. Chicago: University of Chicago Press.

Korobeynikov, G., Korobeinikova, L. y Shatskih, V. (2013). Psychophysiological diagnostics of the functional states in wrestlers. International. Journal of Wrestling Science, 3(2), 5-13. https://doi.org/10.1080/21615667.2013.10878983

Labrador, F. J., De la Puente, M. L. y Crespo, M. (1995). Técnicas de control de la activación: relajación y respiración. En F. J. Labrador, J.A. Cruzado y M. Muñoz (eds.), Manual de técnicas de modificación y terapia de conducta, (pp. 367-395). Madrid: Pirámide.

Leguizamo, F., Olmedilla, A., Núñez, A., Verdaguer, F., Gómez-Espejo, V., Ruiz-Barquín, R. y García-Mas, A. (2021). Personality, Coping Strategies, and Mental Health in High-Performance Athletes During Confinement Derived From the COVID-19 Pandemic. Frontiers in Public Health, 8, 924. https://doi.org/10.3389/ fpubh.2020.561198

Li, S., Wu, Q y Chen, Z. (2020). Effects of psychological interventions on the prevention of sports injuries: A meta-analysis. Orthopaedic Journal of Sports Medicine, 8(8). https://doi. org/10.1177/2325967120928325

Lindern, D. (2016). Desenvolvimento de uma intervenção com foco preventivo baseada na terapia cognitivo-comportamental e na psicologia positiva para atletas de futebol adolescentes. (Tesis Dissertação de Mestrado). Portugal: Pontícia Universidade Católica do Rio Grande do Sul.

López, J. M., Torres, M. D., Berengüi, R., Díaz, A., Martínez, A., Morales, V. y García, J. (2012). Rendimiento físico y psicológico en lucha olímpica: predictores del éxito en lucha femenina. Revista de Psicología del Deporte, 28(1), 215-222. https://revistas. um.es/analesps/article/view/140692

McCormick, A., Meijen, C. y Marcora, S. (2018). Effects of a motivational self-talk intervention for endurance athletes completing an ultramarathon. Sport Psychology, 32, 42-50. https://doi. org/10.1123/tsp.2017-0018

Menéndez, J. I. y Fernández-Río, J. (2015). Niveles de ira en practicantes de boxeo y kickboxing: diferencias en función de la disciplina y el nivel deportivo. Cuadernos de Psicología del Deporte, 15(3), 75-86.

Minot, J., Raynal, P., Chabrol, H. y Monié, B. (2019). Pleine conscience dispositionnelle et bien-être émotionnel chez des adolescents pratiquant la boxe. Journal de Thérapie Comportementale et Cognitive, 29(4), 185-191. https://doi.org/10.1016/j. jtcc. 2019.10.002 
Miró-Moyà, S. L., Torregrosa, M., Regüela, S., Pérez-Rivases, A. y Ramis, Y. (2017). Competencias para la planificación de la carrera dual de deportistas de alto rendimiento. Revista de Psicología del Deporte, 26(4), 51-56.

Monteiro, R., Monteiro, D., Torregrossa, M. y Travassos, B. (2021). Career Planning in Elite Soccer: The Mediating Role of Self-Efficacy, Career Goals, and Athletic Identity. Frontiers in Psychology, 12. https://doi.org/10.3389/fpsyg.2021.694868

Moreno-Fernández, I. M., Gómez-Espejo, V., Olmedilla-Caballero, B., Ramos, L. M., Ortega, E. y Olmedilla, A. (2019). Eficacia de un programa de preparación psicológica en jugadores jóvenes de fútbol. Revista de Psicología Aplicada al Deporte y al Ejercicio Físico, 4(2), Artículo e14. https://doi.org/10.5093/rpadef2019a13

Mostaan, M., Sanatkaran, A. y Bahari, S.M. (2015). The Effects of Behavioral Intervention on Mood in Disabled Professional Basketball Players. Journal of Novel Applied Sciences, 4(7), 797-803.

Oliva, F. J., Calleja, N. y Hernández, M. R. (2012). Escala de creencias sobre la ira en el deporte de combate con atletas mexicanos. Revista Internacional de Medicina y Ciencias de la Actividad Física y del Deporte, 12(45), 110-121. http://cdeporte.rediris.es/ revista/revista45/artescala276.htm

Olmedilla, A. y Dominguez-Igual, J. (2016). Entrenamiento psicológico para la mejora de la atención y la autoconfianza en un futbolista. Revista de Psicología Aplicada al Deporte y al Ejercicio Físico, 1, Artículo e4. https://doi.org/10.5093/rpadef2016a4

Olmedilla, A., Moreno-Fernández, I.M., Gómez-Espejo, V., Robles-Palazón, F.J., Verdú, I. y Ortega, E. (2019). Psychological intervention program to control stress in youth soccer players. Frontiers in Psychology, 10. https://doi.org/10.3389/ fpsyg.2019.02260

Olmedilla, A., Rubio, V. J., Ortega, E. y García-Mas, A. (2017). Effectiveness of a stress management pilot program aimed at reducing the incidence of sports injuries in young football (soccer) players. Physical Therapy in Sport, 24, 53-59. https://doi. org/10.1016/j.ptsp.2016.09.003

Olmedilla, A., Sánchez-Aldeguer, M., Almansa, C., Gómez-Espejo, V. y Ortega, E. (2018). Entrenamiento psicológico y mejora de aspectos psicológicos relevantes para el rendimiento deportivo en jugadoras de fútbol. Revista de Psicología Aplicada al Deporte y al Ejercicio Físico, 3(1), Artículo e8. https://doi.org/10.5093/ rpadef2018a2

Olmedilla-Caballero, B., Moreno-Fernández, I.M., Gómez-Espejo, V. y Olmedilla, A. (2020). Preparación psicológica para los Juegos Paralímpicos y afrontamiento de lesión: un caso en taekwondo. Revista de Psicología Aplicada al Deporte y el Ejercicio Físico, 5(1), e2, 1-13. https://doi.org/10.5093/rpadef2020a4

Pain, M., Harwood, C. y Anderson, R. (2011). Precompetition imagery and music: The impact on flow and performance in com- petitive soccer. The Sport Psychologist, 25(2), 212-232. https:// doi.org/10.1123/tsp.25.2.212

Perez-Rivases, A., Pons, J., Regüela, S., Viladrich, C., Pallarès, S. y Torregrossa, M. (2020). Spanish female student-athletes' perception of key competencies for successful dual career adjustment. International Journal of Sport and Exercise Psychology. https://doi.org/10.1080/1612197x.2020.1717575

Romero, I., Dopico, H. M., Montoro, R., Chávez, E. y Contreras, W. T. (2019). Análisis integral de la motivación en boxeadores. Revista Cubana de Investigaciones biomédicas, 38(2), 56-72. http:// www.revibiomedica.sld.cu/index.php/ibi/article/view/276

Rumbold, J. L., Fletcher, D., y Daniels, K. (2012). A systematic review of stress management interventions with sport performers. Sport, Exercise and Performance Psychology, 7(3), 173193. https://doi.org/10.1037/a0026628

Schinke, R.J. (2004). The contextual side of professional boxing: one consultant's experience. Athletic Insight, 6(2), 1-9.

Schinke, R.J., Stambulova, N.B., Trepanier, D. y Oghene, O. (2015). Psychological support for the Canadian Olympic Boxing Team in meta-transitions through the National Team Program. International Journal of Sport and Exercise Psychology, 13(1), 74-89. https://doi.org/10.1080/1612197X.2014.959982

Schultz, J. H. (1980). El entrenamiento autógeno: Autorrelajación concentrativa. Barcelona: Científico Médica.

Stambulova, N., Engström, C., Franck, A., Linnér, L. y Lindahl, K. (2015) Searching for an optimal balance: Dual career experiences of Swedish adolescent athletes. Psychology of Sport \& Exercise, 21(1), 4-14. htps://doi.org/10.1016/j.psychsport.2014.08.009

Suárez-Rodríguez, M., Soler-Prieto, Y. y Cañizares, M. (2018). Técnicas de intervención psicológica para elevar la concentración de la atención en boxeadores cubanos. Lecturas: Educación Física y Deportes, 22(238), 2-13.

Tessitore, A., Capranica, L., Pesce, C., De Bois, N., Gjaka, M., Warrington, G., MacDonncha, C. y Doupona, M. (2021). Parents about parenting dual career athletes: A systematic literature review. Psychology of sport and exercise, 53, Artículo 101833. https://doi.org/10.1016/j.psychsport.2020.101833

Todd, J. J., Han, S.W., Harrison, S. y Marois, R. (2011). The neural correlates of visual working memory encoding: A time-resolved fMRI study. Neuropsychologia, 49(6), 1527-1536.

Zamora-Martínez, E.A., Rubio, V. y Hernández-López, J.M. (2017). Intervención psicológica para el control de la ansiedad en un deportista español de lucha grecorromana. Acción Psicológica, 14(2), 211-224. https://doi.org/10.5944/ap.14.1.15785

Ziv, G. y Lidor, R. (2013). Psychological Preparation of Competitive Judokas - A Review. Journal of Sports Science and Medicine, 12(3), 371-380. 